\title{
Evaluation of the diagnostic and predictive power of PCA3 in the prostate cancer. A different best cut-off in each different scenario. Preliminary results
}

\author{
Giuseppe Albino ${ }^{1}$, Ettore Capoluongo ${ }^{2}$, Sandro Rocchetti ${ }^{2}$, Sara Palumbo ${ }^{2}$, Cecilia Zuppi ${ }^{2}$, \\ Ettore Cirillo-Marucco ${ }^{1}$ \\ ${ }^{1}$ Operative Unit of Urology, ASL BAT, Andria, Italy; \\ ${ }^{2}$ Laboratory of Clinical Molecular Biology, Institute of Clinical Biochemistry, Catholic University of Sacred Heart, Roma, Italy.
}

\begin{abstract}
Summary Introduction: Aim of this study is to evaluate the diagnostic performance of PCA3 in patients with indication to perform a new biopsy, according to the histological doubt such as High Grade Prostatic Intraepithelial Neoplasia (HGPIN) or Atypical Small Gland Proliferation (ASAP) or the clinical suspicion.

Materials and Methods: One hundred men were enrolled. We used the PCA3 - PROGENSA ${ }^{\mathrm{TM}}$ procedure. After the PCA3 test a repeated prostate biopsy was proposed. The histological findings were correlated to the PCA3 scores. We calculated the positive predictive value (PPV), the sensibility, the specificity, the Youden's index, the ROC curves, the area under the curve (AUC) for each cut-off value of PCA3 score.

Results: These results are preliminary, because at present only 50 of the 100 enlisted men were subjected to rebiopsy. We calculated the best cut-off PCA3 score 20 at the first diagnosis; for patients with HGPIN or ASAP at first biopsy the best sensitivity cut-off is 45; the best cutoff is 45 when you already have a diagnosis of HGPIN, and 35 for ASAP. If we normalize the PCA3 score to the prostate volume, the best cut-off would be 20, with $100 \%$ sensitivity with a prostate volume of $65 \mathrm{ml}$. All results are statistically significant. The real problem, also present in literature, is the constant presence of not diagnosed prostate cancers, for any cut-off value.

Conclusions: Our preliminary results suggest that, to get the best diagnostic performance, it would be wrong to maintain a single cut-off, but it should be chosen according to the scenario of the patients subgroup. It is to explore the possibility to search for the PCA3 in the serum to bridge the gap of the aggressive PCa missed by the urinary test.
\end{abstract}

KEY WORDS: Prostate CAncer gene 3 (PCA3) score; Repeated biopsies; Best cut-off; High Grade Prostatic Intraepithelial Neoplasia (HGPIN); Atypical Small Gland Proliferation (ASAP); Prostate cancer.

Submitted 3 October 2014; Accepted 31 October 2014

\section{INTRODUCTION}

Prostate cancer ( $\mathrm{PCa}$ ) is one of the most common malignancies in the Western world (1). Currently, the early detection of $\mathrm{PCa}$ is mainly based on the digital rectal examination and the increase in PSA levels, which lead to prostate biopsy. Due to the low positive predictive value (PPV) of PSA, that is $18.8 \%$ and $20.2 \%$ based on the cutoff between 2.5 and $10 \mathrm{ng} / \mathrm{ml}$ (2), about $75 \%$ of men with PSA and suspect digital rectal examination (DRE) will be negative at the first prostate biopsy. Even the repeated biopsy will be negative in about $80 \%$ of patients and positive in 20\% (3-5). So when you decide to run a subsequent biopsy, not only the economic aspect has to be taken into account, but also anxiety, discomfort, pain and sometimes severe complications related to diagnostic maneuvers. Therefore, there is the need to identify additional tests that may increase the detection rate of repeated biopsies and reduce the number of unnecessary biopsies. In this regard, the Prostate CAncer gene 3 (PCA3) has shown promising results. Because of the PCA3 gene is overexpressed in $94.6 \%$ of prostate cancers (6) at least 34 times more (x34) compared to non-cancerous prostate tissue (7) and the diagnostic performance of PSA is poor as test for $\mathrm{PCa}$, numerous studies have been performed to evaluate the PCA3 score as a first line in the diagnosis of PCa. The PCA3 test with a score cut-off of 35 results more accurate of the PSA, both with cut-off of $4 \mathrm{ng} / \mathrm{ml}$ and $3 \mathrm{ng} / \mathrm{ml}$ (AUC 0.635 vs 0.550 and 0.581) (2). In addition, lowering the cut-off of the PCA3 score to 20, the accuracy of the test further improves (AUC 0.678) (8). The diagnostic accuracy of the ratio between total PSA/free PSA with $25 \%$ as cut-off is competitive (AUC 0.718 ) to the first diagnosis (9). Therefore for the first prostate biopsy there is no real advantage of PCA3 on PSA. In repeated biopsy, diagnostic accuracy of PSA is reduced, while that of the PCA3 remains constant (10). Aim of this study is to evaluate the performance of PCA3 in patients with indication to perform a repeated biopsy, according to the histological doubt such as High Grade Prostatic Intraepithelial Neoplasia (HGPIN) or Atypical Small Gland Proliferation (ASAP) or the clinical suspicion. 


\section{Materials And Methods}

In order to verify the performance of PCA3 in patients with indication to perform a new biopsy, on the basis of the histological doubt (HGPIN, ASAP) or of clinical suspicion, 100 men were enrolled between September 2011 and June 2013 (21 months) who have already undergone a prostate biopsy the results of which showed the presence of HGPIN or ASAP, or who have already undergone at least 2 prostate biopsies with negative results for $\mathrm{PCa}$, but with high-risk clinical features (suspect DRE, elevated PSA, unfavorable PSA ratio). The characteristics of patients were: 51 patients with HGPIN at the 1st biopsy; 13 with ASAP at the $1^{\text {st }}$ biopsy; 36 with clinical suspicion after $>2$ negative biopsies, mean age 65.8 years (range 48-82), mean PSA $7.86 \mathrm{ng} / \mathrm{dl}$ (range 0.75-33.18) (Table 1). After PCA3 test, a new prostate biopsy has been proposed to the patients. The histological findings of the new biopsies were correlated to the obtained PCA3 score.

\section{Statistical analysis}

We calculated the positive predictive value (PPV), the sensitivity, the specificity, the Youden's index, the ROC curves, the area under the curve (AUC) and statistical reliability (p) for each cut-off value taken in consideration, between 20 and 100. The analysis of the ROC curve by calculating the area under the curve (AUC) measured the diagnostic accuracy of the test. The Youden's index was used to identify the best cut-off (11). For the interpretation of the values of the area under the ROC curve (AUC), you can refer to the classification proposed by Swets (12).

\section{Sampling}

and laboratory procedures

The procedure used is that registered with the brand PROGENSA ${ }^{\mathrm{TM}}$.
Table 1.

Patient characteristics.

\begin{tabular}{|c|c|c|c|c|c|c|c|c|c|}
\hline Nr ident & Iniz & $\begin{array}{c}\text { Bx } \\
\text { before }\end{array}$ & Pre histol & $\begin{array}{c}\text { Current } \\
\text { PSA }\end{array}$ & ratio & $\begin{array}{l}\text { vol } \\
(\mathrm{ml})\end{array}$ & PCA3 & $\begin{array}{l}\text { After Bx } \\
\text { outcome }\end{array}$ & After histol \\
\hline 2 & MG & 2 & phlogosis & 7,75 & $8 \%$ & 17 & 13 & negative & ASAP \\
\hline 1 & SC & 1 & ASAP & 5,55 & $18 \%$ & 78 & 58 & Negative & phlogosis \\
\hline 4 & PV & 2 & phlogosis & 5,74 & $10 \%$ & 48 & 22 & Negative & phlogosis \\
\hline 7 & AM & 2 & phlogosis & 5,8 & $16 \%$ & 108 & 31 & Negative & phlogosis \\
\hline 13 & PM & 2 & phlogosis & 17,85 & $16 \%$ & 108 & 6 & Negative & phlogosis \\
\hline 14 & DSD & 4 & phlogosis & 8,73 & $28 \%$ & 92 & 40 & Negative & phlogosis \\
\hline 15 & MG & 2 & phlogosis & 4,74 & $7 \%$ & 28 & 101 & Negative & phlogosis \\
\hline 20 & RS & 3 & phlogosis & 7,11 & $21 \%$ & 61 & 67 & Negative & phlogosis \\
\hline 21 & $\mathrm{GL}$ & 3 & phlogosis & 30,33 & $16 \%$ & 102 & 36 & Negative & phlogosis \\
\hline 23 & $\mathrm{AV}$ & 2 & phlogosis & 7,93 & $15 \%$ & 105 & 95 & Negative & phlogosis \\
\hline 38 & $\mathrm{TA}$ & 2 & phlogosis & 6,99 & $12 \%$ & 44 & 12 & Negative & phlogosis \\
\hline 39 & $\mathrm{MB}$ & 2 & phlogosis & 5,38 & $16 \%$ & 125 & 39 & Negative & phlogosis \\
\hline 40 & LR & 3 & phlogosis & 9,96 & $20 \%$ & 40 & 46 & Negative & phlogosis \\
\hline 49 & FG & 2 & phlogosis & 9 & $16 \%$ & 105 & 48 & Negative & phlogosis \\
\hline 57 & MV & 2 & phlogosis & 8,22 & $15 \%$ & 88 & 35 & Negative & phlogosis \\
\hline 73 & $P R$ & 2 & ASAP HGPIN & 12,4 & $16 \%$ & 56 & 31 & Negative & phlogosis \\
\hline 78 & $B C$ & 2 & phlogosis & 4,54 & $8 \%$ & 36 & 20 & Negative & phlogosis \\
\hline 82 & $C D$ & 2 & phlogosis & 3,63 & $8 \%$ & 70 & 3 & Negative & phlogosis \\
\hline 87 & $B R$ & 1 & ASAP & 6,6 & $6 \%$ & 44 & 19 & Negative & phlogosis \\
\hline 90 & DAD & 1 & HGPIN & 7,55 & $6 \%$ & 38 & 15 & Negative & phlogosis \\
\hline 93 & DLP & 1 & ASAP & 9 & & 92 & 25 & Negative & phlogosis \\
\hline 99 & $B R$ & 2 & phlogosis & 6,2 & $21 \%$ & 110 & 24 & Negative & phlogosis \\
\hline 6 & SA & 4 & ASAP HGPIN & 5,2 & $28 \%$ & 44 & 19 & Negative & HGPIN \\
\hline 11 & LS & 2 & HGPIN & 9,24 & $62 \%$ & 133 & 44 & Negative & HGPIN \\
\hline 16 & NS & 2 & HGPIN & 10,22 & $16 \%$ & 114 & 12 & Negative & HGPIN \\
\hline 19 & DPP & 2 & phlogosis & 6,01 & $28 \%$ & 56 & 49 & Negative & HGPIN \\
\hline 30 & $\mathrm{BF}$ & 1 & HGPIN & 5,93 & $34 \%$ & 78 & 7 & Negative & HGPIN \\
\hline 44 & $\mathrm{CN}$ & 1 & ASAP HGPIN & 7 & $17 \%$ & 78 & 84 & Negative & HGPIN \\
\hline 45 & PC & 1 & HGPIN & 4,9 & $20 \%$ & 76 & 46 & Negative & HGPIN \\
\hline 50 & $\mathrm{AA}$ & 2 & HGPIN & 12,4 & $14 \%$ & 23 & 29 & Negative & HGPIN \\
\hline 51 & SM & 2 & HGPIN & 6,24 & & 29 & 30 & Negative & HGPIN \\
\hline 74 & $C D$ & 1 & HGPIN & 3,51 & $20 \%$ & 37 & 45 & Negative & HGPIN \\
\hline 79 & $\mathrm{ZF}$ & 2 & HGPIN & 1,82 & $9 \%$ & 13 & 78 & Negative & HGPIN \\
\hline 84 & PS & 2 & phlogosis & 6,76 & $14 \%$ & 44 & 57 & Negative & HGPIN \\
\hline 98 & NM & 1 & HGPIN & 4,02 & $17 \%$ & 28 & 61 & Negative & HGPIN \\
\hline 55 & CG & 3 & ASAP HGPIN & 7,14 & $11 \%$ & 76 & 106 & Negative & HGPIN ASAP \\
\hline 67 & GG & 1 & HGPIN & 5,68 & $19 \%$ & 50 & 52 & Negative & HGPIN ASAP \\
\hline 42 & MF & 2 & ASAP & 4,87 & & 61 & 65 & Positive & GS $5(2+3)$ \\
\hline 27 & GR & 1 & ASAP & 5,62 & $21 \%$ & 50 & 102 & Positive & GS $6(3+3)$ \\
\hline 62 & LA & 1 & HGPIN & 7,9 & $27 \%$ & 29 & 21 & Positive & GS $6(3+3)$ \\
\hline 92 & SA & 1 & ASAP & 6,59 & $7 \%$ & 33 & 11 & Positive & GS $6(3+3)$ \\
\hline 9 & $\mathrm{AF}$ & 1 & ASAP & 26,7 & $18 \%$ & 62 & 163 & Positive & GS $7(3+4)$ \\
\hline 12 & $S R$ & 1 & ASAP HGPIN & 5,87 & $12 \%$ & 45 & 83 & Positive & GS $7(3+4)$ \\
\hline 35 & $\mathrm{GL}$ & 1 & ASAP HGPIN & 7,1 & $14 \%$ & 27 & 172 & Positive & GS $7(3+4)$ \\
\hline 76 & $\mathrm{TL}$ & 3 & phlogosis & 23,3 & $6 \%$ & 17 & 235 & Positive & GS $7(3+4)$ \\
\hline 86 & DGV & 1 & ASAP HGPIN & 13,83 & & 37 & 92 & Positive & GS $7(3+4)$ \\
\hline 3 & DCT & 4 & ASAP HGPIN & 26,78 & $7 \%$ & 30 & 90 & Positive & GS $7(4+3)$ \\
\hline 22 & $\mathrm{GN}$ & 1 & ASAP & 10,92 & $6 \%$ & 22 & 12 & Positive & GS $8(4+4)$ \\
\hline 5 & $\mathrm{MM}$ & 2 & ASAP & 7,07 & $11 \%$ & 87 & 46 & Positive & GS $9(4+5)$ \\
\hline 46 & OG & 1 & HGPIN & 16,21 & $5 \%$ & 38 & 139 & Positive & GS $9(4+5)$ \\
\hline
\end{tabular}

Ident Nr: identification number of the patient's enrollment: Iniz: patient initials; Bx before: number of biopsies performed before the enrollment; Pre histol: histological diagnosis of previous biopsies; Ratio: PSA\%f/t; Vol (ml): prostate volume in ml; PCA3: Detected PCA3 score; After Bx outcome: outcome of the biopsy after the PCA3 test; After histol: histological diagnosis of the biopsy.

\section{RESULTS}

These results are preliminary, because at present only 50 of the 100 enlisted men were subjected to re-biopsy.

The data for these 50 patients are summarized in the Table 1. VPP, sensitivity, specificity and Youden's Index for each cut-off value of the PCA3 score were calculated. The ROC curve of the group of examined patients was obtained (Figure 1A).

The same evaluations were performed for subgroups of patients according to the presence of HGPIN or ASAP: 1) Subgroup of patients who showed HGPIN or PCa (Figure 1B), 2) Subgroup of patients who showed HGPIN in the biopsy performed before or after the PCA3 test (Figure 1C); 3) Subgroup of patients with ASAP at biopsy performed before the PCA3 test (Figure 1D). All 50 patients who underwent re-biopsy after running the PCA3 test were reassessed after we have "normalized" the PCA3 score based on prostate volume that was meas- 


\begin{tabular}{|c|c|c|c|c|}
\hline Cut-off & VPP & sens & spec & Youden's index \\
\hline 20 & 30 & 84,61 & 24,32 & 8,93 \\
\hline 35 & 33,33 & 76,92 & 45,95 & 22,87 \\
\hline 40 & 37,04 & 76,92 & 54,05 & 30,97 \\
\hline 45 & 40 & 76,92 & 59,46 & 36,38 \\
\hline 50 & 47,37 & 69,23 & 72,97 & 42,2 \\
\hline 55 & 50 & 69,23 & 75,68 & 44,91 \\
\hline 60 & 56,25 & 69,23 & 81,08 & 50,31 \\
\hline 65 & 64,29 & 69,23 & 86,49 & 55,72 \\
\hline 70 & 61,54 & 61,54 & 86,49 & 48,03 \\
\hline 100 & 71,43 & 38,46 & 94,59 & 33,05 \\
\hline
\end{tabular}

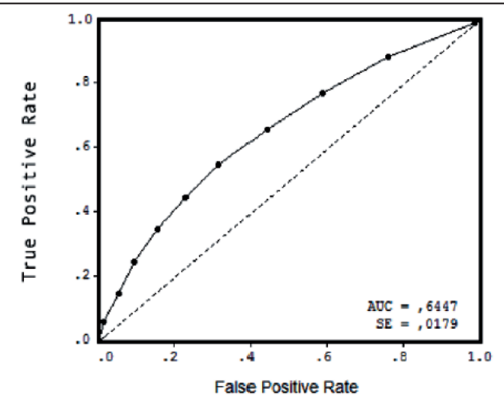

B.

Cut-off sensibility 1-specif Youden's index

\begin{tabular}{|r|r|r|r|}
\hline 20 & $84,61 \%$ & $76,47 \%$ & $8,14 \%$ \\
\hline 35 & $76,92 \%$ & $58,82 \%$ & $18,10 \%$ \\
\hline 40 & $76,92 \%$ & $58,82 \%$ & $18,10 \%$ \\
\hline 45 & $76,92 \%$ & $52,94 \%$ & $23,98 \%$ \\
\hline 50 & $69,23 \%$ & $35,29 \%$ & $33,94 \%$ \\
\hline 55 & $69,23 \%$ & $29,41 \%$ & $39,32 \%$ \\
\hline 65 & $69,23 \%$ & $17,65 \%$ & $51,58 \%$ \\
70 & $61,54 \%$ & $17,65 \%$ & $43,89 \%$ \\
\hline
\end{tabular}

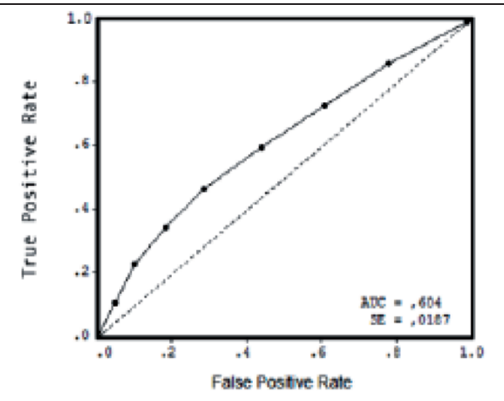

c.

Cut-off sensibility 1-specif Youden's index

$20 \quad 100,00 \% \quad 76,47 \%$

$35 \quad 83,33 \% \quad 58,82 \% \quad 24,51 \%$

$40 \quad 83,33 \% \quad 58,82 \% \quad 24,51 \%$

\begin{tabular}{l|l|l|l}
45 & $83,33 \%$ & $52,94 \%$ & $30,39 \%$
\end{tabular}

$50 \quad 83,33 \% \quad 35,29 \% \quad 48,04 \%$

$55 \quad 83,33 \% \quad 29,41 \% \quad 53,92 \%$

\begin{tabular}{lll|l|}
65 & $83,33 \%$ & $17,65 \%$ & $65,68 \%$ \\
\hline
\end{tabular}

$\begin{array}{llll}70 & 83,33 \% & 17,65 \% & 65,68 \%\end{array}$

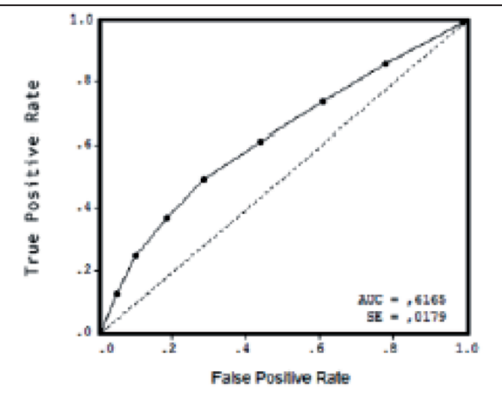

D.

\begin{tabular}{|r|r|r|r} 
Cut-off & sensibility & 1-specif & Youden's index \\
\hline 20 & $80,00 \%$ & $62,50 \%$ & $17,50 \%$ \\
35 & $80,00 \%$ & $37,50 \%$ & $42,50 \%$ \\
50 & $70,00 \%$ & $37,50 \%$ & $32,50 \%$ \\
60 & $70,00 \%$ & $25,00 \%$ & $45,00 \%$ \\
70 & $60,00 \%$ & $25,00 \%$ & $35,00 \%$
\end{tabular}

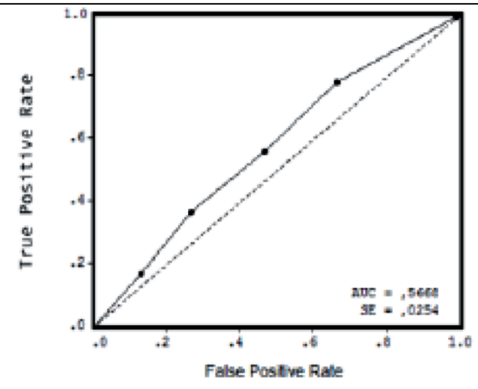

E.

Cut-off sensibility 1-specif Youden's index

$\begin{array}{rrrr}20 & 76,92 & 45,95 & 30,97 \\ 35 & 69,23 & 32,43 & 36,8 \\ 40 & 61,54 & 21,62 & 39,92 \\ 45 & 61,54 & 13,51 & 48,03 \\ 50 & 61,54 & 8,11 & 53,43 \\ 65 & 61,54 & 8,11 & 53,43 \\ 70 & 53,85 & 8,11 & 45,74\end{array}$

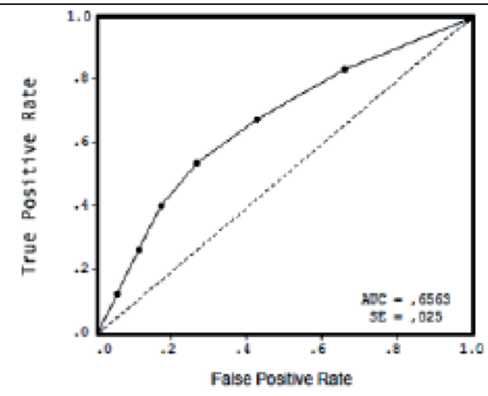

F.

Cut-off Sensibility 1-specif Youden's index

$\begin{array}{rrrr}20 & 100 & 72,41 & 27,59 \\ 35 & 84,61 & 51,35 & 33,26 \\ 40 & 76,92 & 43,24 & 33,68 \\ 50 & 69,23 & 40,54 & 28,69 \\ 65 & 69,23 & 29,73 & 39,5 \\ 70 & 61,54 & 27,03 & 34,51 \\ 100 & 61,54 & 8,11 & 53,43\end{array}$

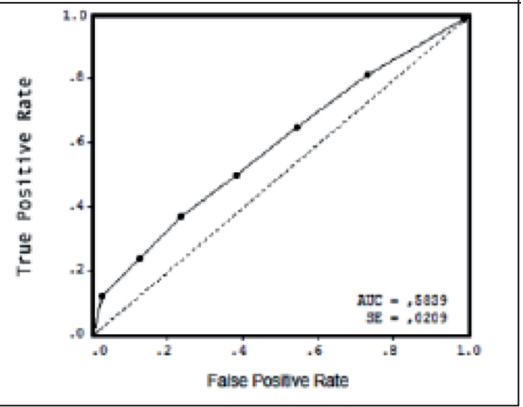

Figure 1.

A. All patients.

B. Subgroup of patients who showed HGPIN or PCA.

C. Subgroup of patients who showed HGPIN in the biopsy performed before or after the PCA3 test.

D. Subgroup of patients with ASAP at biopsy performed before the PCA3 test.

E. PCA3 score based on the volume of prostate gland to a volume of $35 \mathrm{ml}$.

F. РСАЗ score based on the volume of prostate gland to a volume of $65 \mathrm{ml}$.

ured when we collected the urine sample for PCA3, by abdominal ultrasonography (US) of prostate. We started from the following considerations: a prostate of a man over 40 , normally has an average volume of about $35 \mathrm{ml}$; on average, the PCA3 score is considered normal with a cut-off of 35 ; because the PCA3 score is given by the formula PCA3 mRNA/PSA mRNA x1000, it follows that, in case of PCa with a nodule of small volume that partially occupies only one lobe of the prostate, for the same volume of the neoplasm, the score will be inversely proportional to the volume of the prostate, because for the same PCA3 mRNA (same volume of $\mathrm{PCa}=$ same PCA3 mRNA), when greater is the volume of the gland, then greater is also the amount of PSA mRNA present in the urine after the DRE.

Figure 1E shows data evaluation after we "normalized" the PCA3 score based on the volume of the prostate gland to a volume of $35 \mathrm{ml}$. Figure $1 \mathrm{~F}$ shows data evaluation after we "normalized" the PCA3 score based on the volume of the prostate gland to a volume of $65 \mathrm{ml}$. The mean age was 65.8 years and the mean prostate volume $64.7 \mathrm{ml}$, the normalization of the PCA3 score was performed to an average volume of $65 \mathrm{ml}$. Results are summarized in Table 2. 
Table 2. Summary of results.

\begin{tabular}{|l|c|c|c|c|c|c|}
\hline Population under consideration & $\begin{array}{c}\text { Best sensibility } \\
\text { cut-off }\end{array}$ & $\begin{array}{c}\text { Best cut-off } \\
\text { (Youden's index) }\end{array}$ & AUC & $\begin{array}{c}\text { Missed } \\
\text { PCa }\end{array}$ & $\begin{array}{c}\text { Missed } \\
\text { GS }>\mathbf{7}\end{array}$ \\
\hline All patients & 45 & 65 & 0.645 & $\mathrm{p}<0.0001$ & $4 / 13$ & $2 / 4$ \\
\hline Subgroup HGPIN + PCa & 45 & 65 & 0.604 & $\mathrm{p}<0.001$ & $4 / 13$ & $2 / 4$ \\
\hline Subgroup HGPIN before or after PCA3 & 45 & 65 & 0.616 & $\mathrm{p}<0.001$ & $1 / 6$ & $0 / 1$ \\
\hline Soubgroup ASAP & 35 & 35 & 0.567 & $\mathrm{p}<0.01$ & $2 / 10$ & $1 / 2$ \\
\hline PCA3 score normalized to 35 ml of prostate vol & 20 & 50 & 0.656 & $\mathrm{p}<0.0001$ & $5 / 13$ & $2 / 5$ \\
\hline PCA3 score normalized to $65 \mathrm{ml}$ of prostate vol & sensibility: $100 \%$ & 100 & 0.584 & $\mathrm{p}<0.001$ & $5 / 13$ & $2 / 5$ \\
\hline
\end{tabular}

\section{Discussion}

The PCA3 score with cut-off of 35 is the one that offers the best balance between sensibility (58\%) and specificity (72\%) (13) in the diagnosis of PCa at the first biopsy. It shows a positive predictive value ranging between $24 \%$ and $54 \%$ with a loss of $32 \%$ of PCa by diagnosis, $4 \%$ of which with Gleason Score (GS) > $7(2,13,14)$. For this reason, in order to reduce the number of missed PCa at the first diagnosis, several authors have proposed the cut-off of $20(2,15)$. With the cut-off of 20, Roobol et al. have verified a reduction of $\mathrm{PCa}$ missed at diagnosis compared to the cut-off of 35 (12.7\% vs $32.9 \%$ ), none of which was GS $>7$. In the REDUCE trial the PCA3 has exceeded the PSA for PCa-specific diagnostic ability on subjects treated with dutasteride. The median PCA3 score in the arm of dutasteride treated patients did not differ from the median of the control arm treated with placebo, both after 2 and after 4 years of treatment (16). Therefore, dustasteride treatment does not affect PCA3 results.

As regards biopsies subsequent to the first it was verified that the diagnostic accuracy of the PCA3 remains constant regardless of the number of biopsies after the first run on the same patient. Haese et al. (10) showed that the AUC of PCA3 in subsequent biopsies remains constant (between 0.651 and 0.667 ) with a cut-off of 35. PCA3 is higher in patients with HGPIN only compared to those without HGPIN: mean score of 47.9 (CI 36.159.8) compared to 31.8 (CI 24.0-39.7) respectively. These data agree with our experience. A "gray area" of PCA3 in predicting the outcome of the biopsies after the first one. Both in the group of all patients and in subgroups with HGPIN is indicated a cut-off of 45 to achieve the best sensibility of the test and a balanced cut-off of 65 , just to emphasize the confidence intervals indicated by Haese when the HGPIN is present. This is because in our experience more than $50 \%$ of patients undergoing the second (or subsequent) biopsy had a diagnosis of HGPIN in the precedent biopsies.

The fact that patients with HGPIN show a higher mean PCA3 score is found also by other Authors (17). In fact, there are already present many genetic changes in the cells of PIN lesions. The most frequent concern the increased expression of chromosomes $7 p, 7 q, 8 q$ and inactivation of chromosomes 8p, 10q, 13q, 16q and 18q; the inactivation of suppressor genes including the PTEN and the overexpression of c-myc and bcl-2 oncogenes, which play an important role in the initiation and progression of PCa (18). Bussemakers et al. (6) were the first who identified and characterized the DD3 (PCA3) gene comparing PCa tissues containing areas with non-malignant prostate tissue. Since the identification was performed by immunohistochemistry on tissue samples of prostate removed for PCa it is possible that "non-malignant" areas also contained HGPIN that, as already said, is present in $82 \%$ of prostates with PCa. It is therefore likely, that the results of de Kok et al., which showed that the PCA3 was overexpressed x 34 times in malignant tissue and $x 6$ times in non-malignant tissue, are likely to correlated to the presence of HGPIN in the "non-malignant" tissue of a prostate affected by $\mathrm{CaP}(7)$.

The above reasons justified the fact that the best cut-off, identified by Youden's Index, in a subpopulation of patients who are already at their second or subsequent biopsy, can be placed between 45 and 65 , just for the presence of HGPIN in more than half of the cases.

A different matter concerns the subgroup of patients who underwent re-biopsy for the presence of ASAP. The term ASAP was originally used by Iczkowski et al. (19) It refers to minutes tumoral foci: small lesions that disappear in other histological sections, or when cytologic categorical criteria to establish a diagnosis of carcinoma are absent. It may represent an only marginally sampled tumor or one of several benign lesions that mimic malignancy. In practice, it is a "non-diagnosis". In our experience, the best cut-off of the PCA3 test performed only on patients with isolated ASAP (without PIN) at the first biopsy has confirmed the score of 35. Probably because a missed-diagnosis to the first biopsy, restarts the diagnostic path from "time zero".

The scientific literature has now largely confirmed that the PCA3 score is not affected by stage and aggressiveness of the disease, but it is certainly correlated to the volume of the disease (15). Because the formula for the PCA3 score is PCA3 mRNA/PSA mRNA $x 1000$, to say that the PCA3 score is influenced by the volume of the tumor is a partial affirmation, because it affects only the numerator of the formula. Logically, one senses that the PCA3 score should be correlated in direct proportion to the volume of the tumor (PCA3 mRNA in the numerator) and inversely proportional to the volume of prostate without tumor (PSA mRNA in the denominator). For this reason it was also carried out an assessment of the diagnostic appropriateness of the test after the normalizing of the score according to the prostate volume of each patient. In a first case the result was normalized to prostate of $35 \mathrm{ml}$ in average; in a second case has been carried out the normalization of 
the scores computed on a prostate of $65 \mathrm{ml}$ in average (given that the mean age of the subjects was 65.8 years, and the prostate at that age was of $65 \mathrm{ml}$ in average).

Normalizing the score for volume of $35 \mathrm{ml}$ the gray area moves toward the cut-off of 20, as before the first biopsy, and we also get the best result with AUC 0.657. Normalizing the score for prostate volume of $65 \mathrm{ml}$, with the cut-off of 20 it will reach the sensibility of $100 \%$, whereas the best compromise between sensitivity and specificity is obtained with the cut-off of 100 .

The real problem of the diagnostic tests in the decision path to decide on the opportunity to perform a prostate biopsy is the number of PCa that may escape diagnosis according to the used cut-off (the problem exists both for the PSA that for the PCA3, that for all other experimental tests). The literature reports the rate of PCa escaped the diagnosis using PCA3 test: of $12.7 \%$ with a cut-off of 20 and $32.9 \%$ with a cut-off of 35 at the first biopsy; while it is $14.3 \%$ with both cut-off of 20 that with cut-off of 35 in biopsies subsequent to the first one (2). The $36.4 \%$ of $\mathrm{PCa}$ escaped the diagnosis of biopsies following the first one consists of PCa with GS > 7. Even in our series there are potentially missed PCa: between $17 \%$ and $38 \%$. We verified the best results in the subgroup composed exclusively of HGPIN in which there is a risk of $17 \%$ to not diagnose a $\mathrm{PCa}$, but none of $\mathrm{PCa}$ escaped at diagnosis would have had a GS $>7$. The reason why it is possible that more aggressive tumors may escape the diagnosis of PCA3 test resides entirely in the sampling procedure of the diagnostic test. The collected sample has a chance to contain only the cancer cells that are shed in the urine during the DRE. For this to happen it is necessary that the glands, even when they are affected by cancer, continue to be open to the side of the urethra. It is possible, therefore, that the glands with malignancy are no longer open towards the urethra and that the tumor cells, while still producing PCA3 mRNA, are no longer discharged into the urinary stream making the test falsely negative.

\section{Conclusions}

The PCA3 test is a useful diagnostic tool able to guide the decision-making in the early diagnosis of prostate cancer. To get the best diagnostic performance should be considered different cut-off based on biopsy session, prostate volume, histology of initial biopsy. Our results, although preliminary because taken from the 50\% of enrolled men, support that different PCA3 cut-off should be used: 20 at the first diagnosis, 35 in isolated ASAP, 45 in isolated HGPIN. A special focus deserves the opportunity to normalize the score to prostate volume.

\section{RefEREnCes}

1. Siegel $R$, De Santis C, Virgo $K$, et al. Cancer treatment and survivorship statistics, 2012. CA Cancer J Clin. 2012; 62:220-41.

2. Roobol MJ, Schröder FH, van Leeuwen P, et al. Performance of the prostate cancer antigen 3 (PCA3) gene and prostate-specific antigen in prescreened men: exploring the value of PCA3 for a first-line diagnostic test. Eur Urol. 2010; 58:475-81.

3. Seitz C, Palermo S, Djavan B. Prostate biopsy. Minerva Urol Nefrol. 2003; 55:205-18.
4. Raja J, Ramachandran N, Munneke G, Patel U. Current status of transrectal ultrasound-guided prostate biopsy in the diagnosis of prostate cancer. Clin Radiol 2006; 61:142-153.

5. Heidenreich A, Bastian PJ, Bellmunt J, et al. EAU Guidelines on Prostate Cancer. Part 1: Screening, diagnosis, and local treatment with curative intent - Update 2013. Eur Urol. 2014; 65:124-37.

6. Bussemakers MJ, van Bokhoven A, Verhaegh GW, et al. DD3: a new prostate-specific gene, highly overexpressed in prostate cancer. Cancer Res. 1999; 59:5975-9.

7. de Kok JB, Verhaegh GW, Roelofs RW, et al. DD3 (PCA3), a very sensitive and specific marker to detect prostate tumors. Cancer Res. 2002; 62:2695-8.

8. Pepe P, Fraggetta F, Galia A, et al. PCA3 Score and Prostate Cancer Diagnosis at Repeated Saturation Biopsy. Which cut-off: 20 or 35 ? Int Braz J Urol. 2012; 38:489-95

9. Morote J, Trilla E, Esquena S, et al. The percentage of free prostatic-specific antigen is also useful in men with normal digital rectal examination and serum prostatic-specific antigen between 10.1 and 20 ng/ml. Eur Urol. 2002; 42:333-7.

10. Haese A, de la Taille A, van Poppel H, et al. Clinical utility of the PCA3 urine assay in European men scheduled for repeat biopsy. Eur Urol. 2008; 54:1081-8.

11. D'Arrigo G, Provenzano F, Torino C, et al. Diagnostic tests and ROC curves analysis. G Ital Nefrol. 2011; 28:642-7.

12. Swets JA. Measuring the accuracy of diagnostic systems. Science. 1988; 240:1285-93.

13. Auprich M, Bjartell A, Chun FK, et al. Contemporary role of prostate cancer antigen 3 in the management of prostate cancer. Eur Urol. 2011; 60:1045-54.

14. Marks LS, Fradet Y, Deras IL, et al. PCA3 molecular urine assay for prostate cancer in men undergoing repeat biopsy. Urology. 2007; 69:532-5

15. Capoluongo E, Zambon CF, Basso D, et al. PCA3 score of 20 could improve prostate cancer detection: Results obtained on 734 Italian individuals. Clin Chim Acta. 2014; 429:46-50

16. Aubin SM, Reid J, Sarno MJ, et al. Prostate cancer gene 3 score predicts prostate biopsy outcome in men receiving dutasteride for prevention of prostate cancer: results from the REDUCE trial. Urology. 2011; 78:380-5.

17. Galasso F, Giannella R, Bruni P, et al. PCA3: a new tool to diagnose prostate cancer ( $\mathrm{PCa}$ ) and a guidance in biopsy decisions. Preliminary report of the UrOP study. Arch Ital Urol Androl. 2010; 82:5-9.

18. Joniau S, Goeman L, Pennings J, et al. Prostatic intraepithelial neoplasia (PIN): importance and clinical management. Eur Urol. 2005; 48:379-85.

19. Iczkowski KA, MacLennan GT, Bostwick DG. Atypical small acinar proliferation suspicious for malignancy in prostate needle biopsies: clinical significance in 33 cases. Am J Surg Pathol. 1997; 21:1489-95.

\section{Correspondence}

Giuseppe Albino, MD, PhD peppealbino@hotmail.com

Ettore Cirillo-Marucco, MD

Operative Unit of Urology - ASL BAT, Andria, Italy

Ettore Capoluongo, MD - Sandro Rocchetti, MD

Sara Palumbo, MD - Cecilia Zuppi, MD

Laboratory of Clinical Molecular Biology, Institute of Clinical Biochemistry, Catholic University of Sacred Heart, Roma, Italy 